\title{
Initial experience of robotic extrahepatic Glissonean pedicle approach for anatomical liver resection: techniques and short term perioperative outcomes
}

\author{
Jin Ho LEE*, Jae Uk JEONG, Hyung Soon LEE, Kuk Hwan KWON
}

Department of Surgery, National Health Insurance Service Ilsan Hospital, Goyang, Korea

Introduction: Anyone who is a Liver surgeon and performs minimally invasive surgery will try to shorten the surgery time. And we will seek to simplify the operation to make it simpler. In this study, we introduce our initial experience and technique of the extrahepatic Glissonean approach for anatomic liver resections, using a robotic system, and report on short-term perioperative outcomes.

Methods: From August 2016 to December 2020, 10 patients underwent robotic anatomic liver resection using extrahepatic Glissonean approach. The procedure is as follows: (1) mobilization of the liver and isolation and clamping of a selected Glissonean pedicle; (2) transection of the liver parenchyma using a rubber band retraction technique; (3) division of the Glissonean pedicle after full exposure, followed by completion of parenchymal transection.

Results: The median age of the patients was 59.0 (range 42-70) years, and eight were male. Nine patients had hepatocellular carcinoma and the other was liver metastasis. The types of resections performed were as follows: segmentectomy S4 $(\mathrm{n}=1)$, left lateral sectionectomy + segmentectomy S6 $(\mathrm{n}=1)$, right posterior sectionectomy + Wedge resection S2 $(\mathrm{n}=1)$, right hepatectomy $(\mathrm{n}=2)$, left heaptectomy $(n=1)$, bisectionectomy S5 $6(n=2)$, and central bisectionectomy $(n=2)$. The overall complication rate was $20 \%($ grade I, 2; grade II, 0; grade III, 0; grade

Conclusions: Robotic surgery allowed for successful anatomic liver resections via an extrahepatic Glissonean pedicle approach in the right liver and can be safely performed in selected patients. 